\title{
Knowledge of folic acid and spina bifida among female college students and employees in Asir region, Saudi Arabia.
}

\author{
Ibrahim Alnaami ${ }^{*}$, Eman Alayad ${ }^{2}$, Hajer Alamer $^{2}$, Enas Alshashaa ${ }^{2}$, Asma Alahmari ${ }^{2}$, Dhuha \\ Motlag $^{2}$, Fatima Alshehri ${ }^{2}$, Huda Alhashem ${ }^{2}$, Shoug Alkhursan ${ }^{2}$, Majed Aldehri ${ }^{3,4}$, Nabil J Awadalla ${ }^{5}$ \\ ${ }^{1}$ Neurosurgery Section, Department of Surgery, King Khalid University, Abha Maternity and Children's Hospital, Asir \\ Central Hospital, Abha, Saudi Arabia \\ ${ }^{2}$ Department of Surgery, King Khalid University, Abha, Saudi Arabia \\ ${ }^{3}$ Department of Anatomy, King Khalid University, Abha, Saudi Arabia \\ ${ }^{4}$ Department of Neurosurgery and Translational Neuroscience, Maastricht University, Netherlands \\ ${ }^{5}$ Family and Community Medicine Department, King Khalid University, Abha, Saudi Arabia
}

\begin{abstract}
Background: Spina bifida is one of the most challenging and partially preventable congenital diseases that can be prevented with adequate amounts of folic acid (FA) intake before conception and throughout the first few months of pregnancy. Knowledge about the adequate timing and duration of FA intake is critical for prevention.

Methods: A questionnaire-based, cross-sectional study was conducted to assess spina bifida and FA awareness among female students at King Khalid University (KKU), Abha, Saudi Arabia. The study included three groups: health-college female students (HCS), non-health-college female students (NHCS), and KKU female employees (others). These three groups were attendants at the Spina Bifida Awareness Week that was held at KKU in March 2017.

Results: There were 1,366 participants, of whom $11 \%$ were HCS, $59 \%$ were NHCS, and $30 \%$ were "others". Across all tested domains (i.e., spina bifida awareness, FA intake time and duration, diseases prevented by folic acid intake, and food rich in folic acid), it was found that statistically significant differences existed. The results showed a highly significant lack of knowledge towards the aforementioned domains in both the NHCS and "others" groups. Marital status and number of children were independent variables and showed statistically significant differences in knowledge toward the tested domains.

Conclusion: In Saudi Arabia, more attention should be paid to educate women of childbearing age on the importance of FA intake in preventing spina bifida. These results indicate how FA intake awareness should be embedded in junior-high and secondary school curricula to further educate females on the importance of FA.
\end{abstract}

Keywords: Folic acid, Spina bifida, Awareness, Saudi Arabia

Accepted on August 30, 2018

\section{Introduction}

The management of myelomeningocele (MMC), also known as spina bifida, remains a challenge for all medical providers [1]. Preventing this disorder is possible via food fortification and adequate amounts of folic acid (FA) intake before conception and throughout the first month of pregnancy. However, this preventive method is not effective for hereditary types of myelomeningocele [2].

Multiple studies [3] have been conducted over the last decade to explore the prevalence of MMC in Saudi Arabia; however, the numbers have not significantly changed between 1997 and 2009, with prevalence rates ranging from 0.8-1.46 [4].

This is in spite of policy developments surrounding FA fortification in some foods, as adopted in Saudi Arabia in year $2000[4,5]$.

Based on the numbers given in previous studies, 780 children are expected to be born with MMC in Saudi Arabia each year [6]. As such, the Ministry of Health in Saudi Arabia adopted a policy whereby all pregnant women are given FA during pregnancy; however, one of the main problems faced is that 
women end up taking FA after the most critical period (i.e., at w 4-8 of pregnancy), at which point neural tube formation takes place [7]. Several studies have shown that despite implementing this policy, whereby FA is provided during pregnancy, only $4 \%-8.3 \%$ of Saudi women actually take FA during pregnancy $[3,8]$.

Kari et al. as well as other researchers, have shown that a high percentage of educated women $(88 \%)$ were unaware of the importance of FA in preventing neural tube defects (NTDs) in western Saudi Arabia [9]. Compared to other western countries, the incidence of NTDs in the Asir region appears to have remained static over the years, a situation which necessitates further investigation and analysis [10].

This study thus aimed to assess female college students and female college employees' knowledge toward FA and spina bifida in the Asir region.

\section{Methods}

This questionnaire-based study was conducted with female college students and employees at King Khalid University, Abha, Saudi Arabia, to evaluate their awareness of FA intake during pregnancy and its relationship with the development of spina bifida. The questionnaire (Figure 1), which asked questions related to the importance of FA intake in preventing NTDs (specifically, spina bifida), was distributed among college students. After obtaining ethical approval from the institutional review board (IRB) at King Khalid University, the study was carried out while maintaining participant confidentiality. The questionnaires included participant demographic-related questions, as well as questions related to the participants' knowledge of FA and its sources. The questionnaire included questions on the optimal time to take FA in order to prevent NTDs, the appropriate dose of FA required, the best sources to get the recommended dose of FA, and the participants' attitudes toward FA intake. The target population was female college students from 8 different women's colleges; there were no exclusion criteria. The educational materials (lecture, leaflets, and posters) were offered to more than 3,000 subjects following data collection; these were part of a regional awareness campaign conducted within the Asir region.

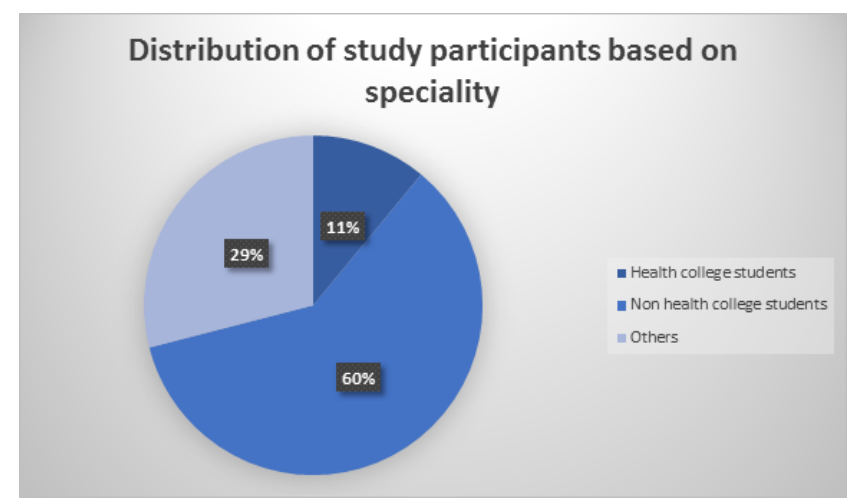

Figure 1. Questionnaire distributed to the participants.
A total of 1,366 participants were involved in our study. A structured data sheet was created to aid in data collection. Data were categorized, coded, and processed with SPSS software (IBM Corporation, Armonk, NY, USA). Continuous variables were expressed using means and standard deviations, and categorical variables were expressed using frequencies.

\section{Results}

The total number of participants was 1,366; participants were divided into three groups: 146 were in the HCS group, 806 were in the NHCS group, and 414 were in the "others" group. Details related to education level, marital status, and other variables are presented in Table 1 and Figure 2.

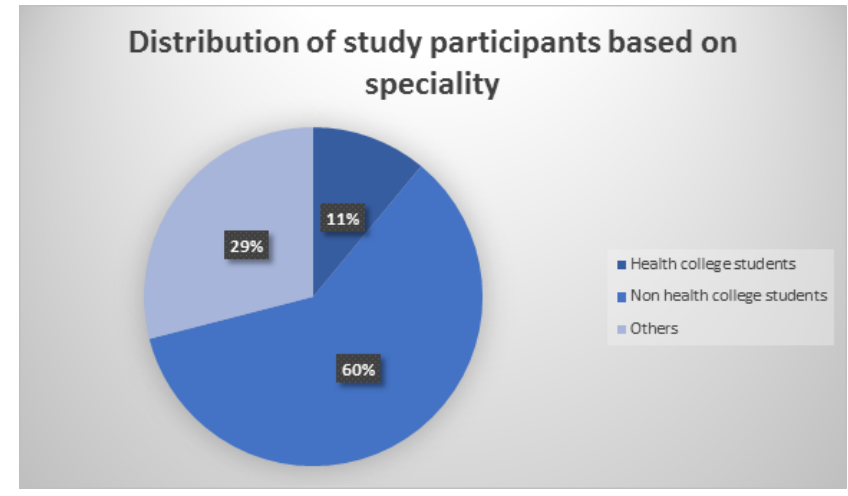

Figure 2. Pie chart of participant distribution.

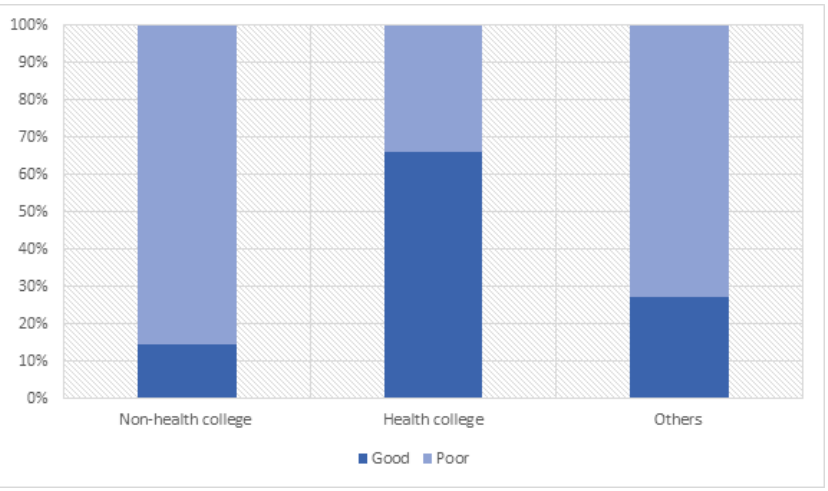

Figure 3. Bar chart showing the overall score of FA knowledge across the 3 groups.

Participants' mean overall knowledge scores related to FA and spina bifida were low in all three groups; however, the HCS group had a relatively higher score $(\mathrm{P}<0.0001)$.

When exploring the questionnaire results further, it was found that only $34 \%$ of those in the NHCS group were aware of FA, $35 \%$ knew that FA should be used during pregnancy, while only $14 \%$ had heard of spina bifida, and only $7 \%$ knew of foods rich in FA.

Participants' knowledge on the duration of FA use during pregnancy was $58 \%$ among those in the HCS group and $29 \%$ for those in the "others" group; this difference was highly significant $(\mathrm{P}<0.0001)$. 
The question on when women should start taking FA was unfortunately answered poorly, even among those in the HCS group, where only $16 \%$ of participants provided a correct answer-specifically, that FA must be used before conception. Similar results were seen among those in the NHCS and "others" group at $15 \%$ and $14 \%$, respectively $(\mathrm{P}=0.75)$.

It was found that $29 \%$ of those in the NHCS group and $25 \%$ of those in the "others" group provided correct responses related to the preventive role of FA. Moreover, $33 \%$ of those in NHCS group $28 \%$ of those in the "others" group correctly identified the FA dose required during pregnancy.

Among the participants in this study, $49 \%$ of those in the NCHS group provided correct statements related to when and how long to take FA, while $58 \%$ of those in the HCS group and $33 \%$ of those in the "others" group provided correct responses on this same topic $(\mathrm{P}<0.0001)$.

We investigated whether having children or not had an impact on FA knowledge. It was generally found that there was no significant difference between those who had children versus those who did not across the various FA-related domains, such as FA intake timing and knowledge of FA-rich foods. However, having a disabled child or relative was significantly associated with FA knowledge across all domains. In terms of numbers, the level of knowledge about the importance of FA intake among those with children was $49.4 \%$ compared to $46.4 \%$ of those without children $(\mathrm{P}=0.44)$ (Table 2$)$. Moreover, $55 \%$ of participants who had children, were aware of FA intake timing, while $56 \%$ of females who do not have children, were aware of this information $(\mathrm{P}=0.57)$. Further, $37 \%$ of women with children were knowledgeable about FA-rich foods, whereas $32 \%$ of women without children were aware of the same $(\mathrm{P}=0.2)$ (Figure 3$)$.

Again, when exploring whether having children or not had an impact on FA knowledge, it was found that only $9.7 \%$ of subjects with children were aware of diseases prevented by FA, whereas $4.9 \%$ of subjects without children were aware of these diseases $(\mathrm{P}=0.01)$. Conversely, $10.8 \%$ of those with a disabled child or relative and $7.1 \%$ of those without a disabled child or relative had prior knowledge of FA deficiency-related diseases $(\mathrm{P}=0.05)$.

Moreover, participants' knowledge of spina bifida was also assessed and the results revealed that only $22.6 \%$ and $26.8 \%$ of subjects with children and those with a disabled child or relative, respectively, were aware of this birth defect; this difference was significant $(\mathrm{P}=0.04)$.

The level of all FA-related knowledge was remarkably different when comparing women with a child or relative with a congenital disability versus those without. Specifically, participants' awareness of the importance of FA among those without a disabled child or relative was $33.8 \%$, as compared to $47.4 \%$ of those with a disabled child or relative $(\mathrm{P}=0.001)$. Also, the participants' general knowledge of spina bifida was $29 \%$ and $16.9 \%$ among women without disabled children versus those with a disabled child or relative, respectively $(\mathrm{P}=0.001)$. Across all other domains of FA-related knowledge, highly significant differences were found (Table 3 ).

Table 1. Socio-demographic characteristics of the sampled females according to specialty.

\begin{tabular}{|c|c|c|c|c|c|c|c|c|c|}
\hline \multirow[t]{3}{*}{ Characteristics } & & \multicolumn{6}{|c|}{ Group } & \multicolumn{2}{|l|}{ Total } \\
\hline & & \multicolumn{2}{|c|}{ Health college } & \multicolumn{2}{|c|}{ Non-health college } & \multicolumn{2}{|c|}{ Others } & \multirow[b]{2}{*}{ No } & \multirow[b]{2}{*}{$\%$} \\
\hline & & No & $\%$ & No & $\%$ & No & $\%$ & & \\
\hline \multirow[t]{2}{*}{ Age in years } & $<20 y$ & 19 & $13.00 \%$ & 154 & $19.10 \%$ & 236 & $56.90 \%$ & 409 & $29.90 \%$ \\
\hline & $>20 y$ & 127 & $87 \%$ & 652 & $80.90 \%$ & 179 & $40.30 \%$ & 958 & $70.10 \%$ \\
\hline \multirow[t]{2}{*}{ Marital status } & Single & 76 & $52.10 \%$ & 408 & $50.60 \%$ & 247 & $59.50 \%$ & 731 & $53.50 \%$ \\
\hline & Married & 70 & $47.90 \%$ & 398 & $49.40 \%$ & 168 & $40.50 \%$ & 636 & $46.50 \%$ \\
\hline \multirow[t]{2}{*}{ Have children } & No & 58 & $82.90 \%$ & 328 & $82.40 \%$ & 149 & $88.70 \%$ & 535 & $84.10 \%$ \\
\hline & Yes & 12 & $17.10 \%$ & 70 & $17.60 \%$ & 19 & $11.30 \%$ & 101 & $15.90 \%$ \\
\hline \multirow[t]{2}{*}{ Children with anomaly } & Yes & 2 & $16.70 \%$ & 22 & $31.40 \%$ & 4 & $21.10 \%$ & 28 & $27.70 \%$ \\
\hline & No & 10 & $83.30 \%$ & 48 & $68.60 \%$ & 15 & $78.90 \%$ & 73 & $72.30 \%$ \\
\hline \multirow[t]{2}{*}{ Have relative with congenital anomaly } & Yes & 36 & $24.70 \%$ & 157 & $19.50 \%$ & 80 & $19.30 \%$ & 273 & $20.00 \%$ \\
\hline & No & 110 & $75.30 \%$ & 649 & $80.50 \%$ & 335 & $80.70 \%$ & 1094 & $80.00 \%$ \\
\hline
\end{tabular}

Table 2. Relation of folic acid knowledge with presence of children among married group and presence of disabled child and or relative among study group. 


\begin{tabular}{|c|c|c|c|c|c|c|}
\hline & Yes $(n=557)$ & No $(n=328)$ & No $(n=1173)$ & Yes $(n=194)$ & & \\
\hline Folic acid (FA) importance & $275(49.4)$ & $153(46.6)$ & 0.44 & $396(33.8)$ & $92(47.4)$ & 0.001 \\
\hline Spina bifida & $126(22.6)$ & $95(29.0)$ & 0.04 & $198(16.9)$ & $52(26.8)$ & 0.002 \\
\hline FA intake timing & $302(54.2)$ & $185(56.4)$ & 0.57 & $512(43.6)$ & $110(56.7)$ & 0.001 \\
\hline FA intake duration & $284(51.0)$ & $139(42.4)$ & 0.01 & $383(32.7)$ & $110(56.7)$ & 0.001 \\
\hline Diseases prevented by FA & $54(9.7)$ & $16(4.9)$ & 0.01 & $83(7.1)$ & $21(10.8)$ & 0.05 \\
\hline Food rich in FA & $207(37.2)$ & $107(32.6)$ & 0.19 & $364(31.0)$ & $78(40.2)$ & 0.01 \\
\hline
\end{tabular}

Table 3. Participants' awareness regarding folic acid and spina bifida.

\begin{tabular}{|c|c|c|c|c|c|c|c|c|c|}
\hline & \multirow{2}{*}{$\begin{array}{l}\text { Health } \\
(n=146\end{array}$} & \multirow{2}{*}{$\begin{array}{l}\text { college } \\
\%\end{array}$} & \multirow{2}{*}{ students } & \multicolumn{2}{|c|}{$\begin{array}{l}\text { Non-health } \\
(n=806)\end{array}$} & \multirow[t]{2}{*}{ students } & \multicolumn{2}{|c|}{ Others $(n=414)$} & \multirow[t]{2}{*}{$P$ value } \\
\hline & & & & No. & $\%$ & & No. & $\%$ & \\
\hline folic acid & 86 & 58.9 & & 276 & 34.2 & & 126 & 30.4 & $<0.001$ \\
\hline Spina bifida & 72 & 49.3 & & 118 & 14.6 & & 60 & 14.5 & $<0.001$ \\
\hline Duration of folic acid use & 85 & 58.2 & & 287 & 35.6 & & 121 & 29.2 & $<0.001$ \\
\hline Foods rich in folic acid & 27 & 18.5 & & 58 & 7.2 & & 19 & 4.6 & $<0.001$ \\
\hline Preventive role of folic acid & 82 & 56.2 & & 235 & 29.2 & & 103 & 24.9 & $<0.001$ \\
\hline Proper dose of folic acid & 32 & 21.9 & & 268 & 33.3 & & 116 & 28 & 0.004 \\
\hline Most correct statement & 85 & 58.2 & & 399 & 49.5 & & 138 & 33.3 & $<0.001$ \\
\hline
\end{tabular}

\section{Discussion}

Despite the abundance of materials and social media-related content on FA, as well as the implementation of awareness programs adopted by health authorities in Saudi Arabia, there seems to be a lack of FA-related knowledge among females in Saudi Arabia, even among those who are highly educated.

Many previous studies conducted in Saudi Arabia, such as those by Al-Akhfash et al. [11] showed that a strikingly low number $(8.3 \%)$ of females who had a previous history of delivering children with congenital anomalies were taking FA during pregnancy. Another study conducted by Alshail et al. [12] who performed their research in one of the major hospitals in Saudi Arabia in 2014, had involved more than 600 patients. The authors found that only $5 \%$ of Saudi females were taking FA during pregnancy. Our study produced similar numbers; however, we expected a higher level of knowledge related to preconception care, especially among highly educated women and in the context of the social media storm and the abundance of information technology. Compared to other studies conducted at the King Khalid University Hospital in Riyadh, our results showed that there was a lack of knowledge of FA and NTDs among Saudi women, even among highly educated college students [13].

Our results were similar to those from another study conducted at King Abdulaziz University Hospital, Jeddah. In that study, hospital patients were interviewed and the researchers found that there were higher rates of knowledge about FA was significantly higher in women at their late reproductive period, and among those who receive higher level of antenatal care, and advanced maternal age in their study population [14].

Moreover, population statistics are also reflective of the low knowledge levels related to FA intake during pregnancy. Specifically, most Saudi females (66\%) reported starting FA when they realized that they were pregnant. By this time, they had passed the critical period of neural tube formation, which is a major concern, especially for unplanned pregnancies [15].

There is a pressing need to educate young females of childbearing age about healthy practices in terms of vitamin and FA intake, even before marriage [16]. In fact, a lack of education and lower rates of planned pregnancy was the main contributors to the static (and low) rates of FA intake among women in Saudi Arabia over the past 12 years [12].

Since 2012, progress has been made in terms of reducing the development rates of FA-preventable spina bifida worldwide, although this rate reduction is still low at 13.2 as per $2015 \%$ [17]. Additional efforts must be made to increase the level of awareness and implementation of highly specialized health education programs to young females. These initiatives should incorporate information on preconception care, especially in terms of the development of NTDs and the associated burden.

An in-depth review by Kelly et al. found that FA awareness and the level of knowledge of the benefits of FA was relatively low among women of childbearing age in the years spanning 1998-2010. The authors found that advanced levels of FA- 
related knowledge were associated with higher education levels and household income [18]. Similarly, Eghwrudjakpo et al. found that amongst women of reproductive age in a tertiary health institution, the level of awareness of the role played by FA in the prevention of NTDs was variable. Specifically, $94.4 \%$ of participants had some knowledge about FA, whereas $29.6 \%$ knew something about NTDs. This reiterates the need to enhance women's awareness of FA-related information to ensure that at-risk populations benefit and reduce their chances of facing NTD development in their offspring [19].

\section{Strengths of the study}

There are multiple strengths of this study. First is the fact that it targeted highly educated women, while many of the previous local studies described earlier targeted the general population. However, in our study, we showed that a gap exists in terms of FA-related knowledge, even among highly educated women, as exhibited by those in the NHCS group (even when compared to the "others" group) and another strength is the large number of study subjects. This investigation is considered to be one of the largest local studies, which increases the generalizability of our results to the general Saudi population.

\section{Limitations of the Study}

The study shares the limitations commonly faced when conducting questionnaire-based studies, such as whether all participants were able to understand all questionnaire items, whether subjects provided truthful answers, and a lack of validity. However, the authors believe that performing the study with highly educated participants, as done here, may have helped to overcome some of the limitations mentioned above.

\section{Conclusion}

There should be greater attention and efforts made to educate women of child bearing age in Saudi Arabia on the importance of FA in preventing spina bifida burden. The results of this study provide compelling evidence in support of a mandate to educate women further by incorporating FA intake-related information in their education curricula in junior-high and secondary school.

\section{References}

1. Addas BM. Split cord malformation type I distal to segmental myelomeningocele. Saudi Med J 2014; 35: 72.

2. Salih MA, Murshid WR, Seidahmed MZ. Classification, clinical features, and genetics of neural tube defects. Saudi Med J 2014; 35: 5.

3. Seidahmed MZ, Abdelbasit OB, Shaheed MM, Alhussein KA, Miqdad AM, Khalil MI. Epidemiology of neural tube defects. Saudi Med J 2014; 35: 29.

4. Al Rakaf MS, Kurdi AM, Ammari AN, Al Hashem AM, Shoukri MM, Garne E. Patterns of folic acid use in pregnant Saudi women and prevalence of neural tube defects-results from a nested case-control study. Prev Med Rep 2015; 2: 572-576.

5. Salih MA. Neural tube defects: challenging, yet preventable. Saudi Med J 2014; 35: 3.

6. Al-Odaib AN, Al-Sedairy ST. An overview of the Prince Salman Center for Disability Research scientific outcomes. Saudi Med J 2014; 35: 75.

7. Al-Holy M, Eideh A, Epuru S, Abu-Jamous D, Ashankyty I. Awareness of Folic Acid Intake among Women in the Childbearing Age in Hail Region-Saudi Arabia. Food Nutr Sci 2013; 4: 49.

8. Al Rakaf MS, Kurdi AM, Ammari AN, Al Hashem AM, Shoukri MM, Garne E. Patterns of folic acid use in pregnant Saudi women and prevalence of neural tube defects-results from a nested case-control study. Prev Med Rep 2015; 2: 572-576.

9. Kari J. Folic acid awareness among female college students: neural tube defects prevention. Saudi Med J $2008 ; 12$.

10. Hamamy H. Epidemiological profile of neural tube defects in Arab countries. Mid East J Med Gene 2014; 3: $1-10$.

11. Al-Akhfash AA, Abdulla AM, Osman AM, Abdulgafar JI, Almesned AA. Maternal knowledge and use of folic acid among Saudi females. Saudi Med J 2013; 34: 1173-1178.

12. Alshail E, De Vol E, Yassen A, Elgamal EA. Epidemiology of neural tube defects in Saudi Arabia. Saudi Med J 2014; 35: 68.

13. Al-Hakeem MMMK. Impact of education on knowledge and use of folic acid among Saudi Women 2012.

14. Bukhari A, Bajouh $\mathrm{O}$, Aljehani $\mathrm{M}$, Alzahrani $\mathrm{N}$, AlQahtani A. The awareness of folic acid supplements among women of childbearing age in King Abdulaziz University Hospital, Jeedah-Saudi Arabia. J Nurs Care 2016; 5: 2167-1168.

15. McWalter P, Al Shmassi A, Eldali A. Awareness and use of folic acid in a clinic-based Saudi pregnant population. Saudi J Medic Med Sci 2015; 3: 141.

16. Fatima A, Saif N, Ghani M, Ali S. Knowledge of women; supplementation of folic acid in periconceptional period. Profess Med J 2016; 23.

17. Youngblood ME, Williamson R, Bell KN, Johnson Q, Kancherla V, Oakley GP. 2012 update on global prevention of folic acid-preventable spina bifida and anencephaly. Birth Defect Res Part A Clin Mol Teratol 2013; 97: 658-663.

18. Fehr KR, Fehr KD, Protudjer JLP. Knowledge and use of folic acid: in women of reproductive age. Canad J Diet Pract Res 2011; 72: 197-200.

19. Eghwrudjakpor P, Amadi C, Amusan E. Evaluation of the level of awareness of the role of folic acid in the prevention of neural tube defects amongst women of reproductive age in a tertiary health institution. Nigerian $\mathrm{J}$ Med J Nat Assoc Resid Doct Niger 2011; 20: 207-212. 


\section{*Correspondence to}

Ibrahim Alnaami

Neurosurgery Section

Department of Surgery

King Khalid University

Abha Maternity and Children's Hospital

Asir Central Hospital

Saudi Arabia 